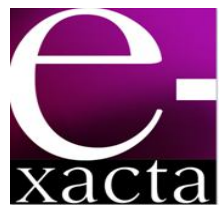

\title{
AUTOMAÇÃO DE UMA PLANTA DE PRODUÇÃO DE BIODIESEL
}

\section{Automation of a Biodiesel Production Plant}

ISSN: 1984-3151

\author{
Marco Aurélio de Souza Birchal'; Viviane Santos Birchal ${ }^{2}$
}

1 Doutor em Engenharia Elétrica. CPDEE - Escola de Engenharia/UFMG, 2005. Professor do Departamento de Engenharia de Controle e Automação - PUCMinas birchal@pucminas.br.

2 Doutora em Engenharia Mecânica. DEMEC - Escola de Engenharia da UFMG, 2003. Professora do Departamento de Engenharia Química - Escola de Engenharia/UFMG. Belo Horizonte, Minas Gerais.vsbirchal@gmail.com.

Recebido em: 20/09/2013 - Aprovado em: 28/11/2013 - Disponibilizado em: 30/11/2013

RESUMO: O Brasil é um grande produtor de álcool largamente utilizado como combustível automotivo. Seu uso na produção de biodiesel cria uma sinergia entre os dois produtos, trazendo oportunidades de novos investimentos $e$ pesquisas em ambas áreas. Apesar do grande potencial que representa, é também verdade que muitas vezes a qualidade do biodiesel é comprometida por métodos de produção rudimentares. O processo de automação emerge como uma poderosa ferramenta na busca de uma maneira de produção melhor e mais confiável. Este trabalho apresenta a automação de uma planta de produção de biodiesel como resultado de uma cooperação entre a PUC - Pontifícia Universidade Católica de Minas Gerais - e a UFMG - Universidade Federal de Minas Gerais -, de modo a aplicar as melhores práticas do estado da automação na produção de biodiesel. Este estudo objetiva manter a auto sustentabilidade e a eficiência do processo de biorefinaria na busca de energia limpa como uma contribuição à matriz energética brasileira. A automação se deu em uma planta em escala piloto, que possibilita a variação de muitos parâmetros, como o tempo e a temperatura de reação e de destilação que, no seu projeto original, necessita de muita interação humana para manter o processo funcionando. Este trabalho emprega tecnologias de automação e controle de última geração, o que resulta em uma maneira robusta, segura e econômica de operar um processo de biorefinaria.

PALAVRAS-CHAVE: Biodiesel. Automação de Processos. Biorefinaria.

ABSTRACT: Brazil raises as one of the most promising countries in use of the biomass energy technology due to plenty of its characteristics, such as the large agriculture production and the diversified oleaginous complex, allowing access of many biomass raw materials. Brazil is also a great producer of alcohol widely used as automotive fuel. The biodiesel necessity of alcohol stimulates its productivity and creates a synergy between them, bringing opportunities of new investments and researches. Although the great potential it represents, it is also true that many times the quality of the production is compromised by the archaic and rudimental methods applied. The automation process emerges as a powerful tool in the search of a better and more reliable way of production. This work presents the automation of a pilot biodiesel production plant as a result of cooperation between PUC Minas Pontifical Catholic University of Minas Gerais and UFMG - Federal University of Minas Gerais, Brazil, in a way of applying the best practices of the state of the art of automation in biodiesel production. This work aims on keeping the auto sustainability and efficiency of the biorefinery process in the search of clean energy as a contribution to the Brazilian energetic matrix. The plant is a self contained pilot scale of an industrial plant that gives the possibility of many parameters variation, like time and temperature reaction and distillation that, in its original design, needs much human interaction to keep the process running. By the use of modern automation devices, this work redesigned the controller process in a high and confident level of automation. As the result, the totally redesigned control process gives a reliable, secure and economic way of operating a biorefinery process.

KEYWORDS: Biodiesel;Biodiesel. Process Automation. Biorefinery. 


\section{INTRODUÇÃO}

O governo brasileiro, através do PNPB - Programa Nacional de Produção e Uso do Biodiesel -, lançado em 2004, estabeleceu o biodiesel como um dos elementos da matriz energética brasileira. Desde então, e cada vez mais, a importância deste combustível se faz presente, principalmente após 2008, quando, por força de lei, instituiu-se a obrigatoriedade da presença de $2 \%$ (B2) de biodiesel no diesel de petróleo distribuído no país. Este percentual aumentou para 5\% (B5) em 2010 (MINISTÉRIO DE MINAS E ENERGIA, 2013).

A despeito da obrigatoriedade legal da adição do biodiesel ao combustível convencional e dos investimentos da indústria em sua obtenção, observase um baixo emprego de tecnologias de ponta e uma consequente ineficiência nos meios de produção do biodiesel. Esta em muito se dá pelo modesto emprego da automação de processos nas plantas de obtenção de biodiesel. A correta automatização do processo pode levar uma planta semiautomática a níveis de produção muito superiores, sem a necessidade de alterações substanciais em seus equipamentos de processo.

Somente com a compreensão do processo de produção do biodiesel e da identificação de seus elementos constituintes pode-se chegar a uma proposta efetiva de melhoria da produção deste combustível através da interferência em certas etapas desse processo.

Este estudo, no entanto, se mostra interdisciplinar e exige tanto uma análise por parte da engenharia química, detentora do conhecimento do processo, quanto da engenharia de controle e automação, responsável por achar os meios de automatizar os equipamentos de processo envolvidos.
O presente trabalho apresenta a execução desse estudo e sua posterior implementação em uma planta piloto de biodiesel. $\mathrm{O}$ trabalho foi desenvolvido através da cooperação entre o Departamento de Controle e Automação da Pontifícia Universidade Católica de Minas Gerais e o Departamento de Engenharia Química da Universidade Federal de Minas Gerais, em um processo de produção de biodiesel em escala piloto, sem nenhum nível de automação.

Aqui trata-se os aspectos técnicos da planta e os meios usados para torná-la um processo automatizado de produção.

\section{A Planta Piloto}

A planta piloto de uma biorefinaria (BIOMINAS, 2012) utilizada neste trabalho é um modelo em escala de uma planta de produção industrial de biodiesel que apresenta seu sistema reacional e de processamento capaz de simular as condições e características de processos industriais reais, de modo a fornecer conhecimento no controle das variáveis de processo principais, tais como a natureza do óleo vegetal, álcool e catalisador empregados, tempo e temperatura de reação de destilação.

\subsection{Produção de Biodiesel na Planta}

Na produção do biodiesel, é necessário utilizar óleo vegetal purificado ou reciclado obtido através de tratamento, metanol ou etanol anídrico e um catalisador alcalino. A FIG 1 apresenta o processo em que o destilador e o reator são aquecidos em tanques reservatórios (BIOMINAS, 2012). 


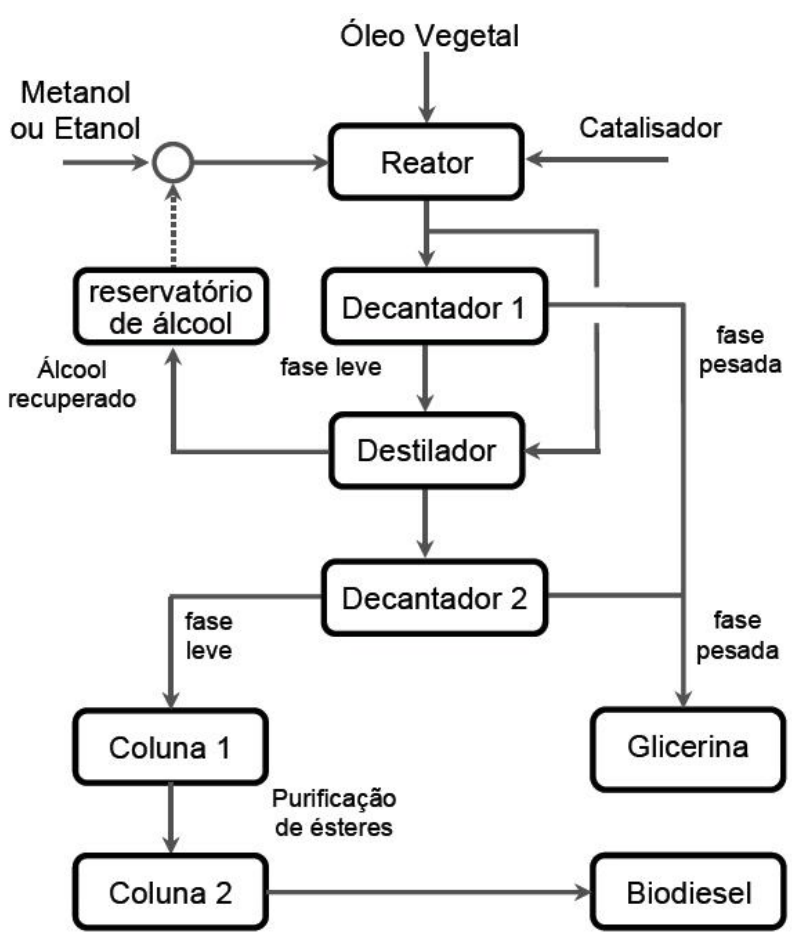

FIGURA 1 - Fluxograma do processo

A transesterificação, reação química envolvida na produção de biodiesel, ocorre no reator, onde a mistura de álcool (metanol ou etanol), catalisador $(\mathrm{NaOH}, \mathrm{KOH}$ ou metóxido de sódio $30 \%)$ e óleo vegetal é realizada. Então, os triglicerídeos (óleo e graxa) são convertidos em ésteres (biodiesel) e glicerina (como um subproduto). A FIG. 2 representa a reação química (DRAPCHO; NGHIEM; WALKER, 2008).

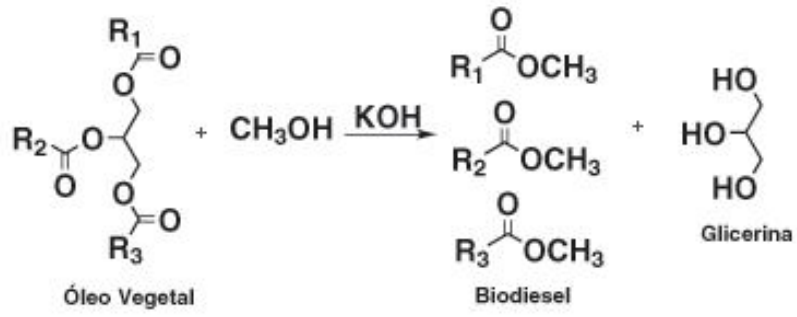

FIGURA 2 - Reação de transesterificação

\subsection{Operação da Planta}

A FIG. 3 representa as partes principais da planta. O processo de produção do biodiesel inicia-se com a adição de óleo vegetal no reator (1) com álcool e catalisador, sob aquecimento controlado e vigorosa agitação para assegurar a mistura das duas fases, por 60 a 120 minutos, de maneira a garantir a conversão máxima em éster. Os resultados do processo são o biodiesel e a glicerina, os quais devem ser separados antes ou após a etapa de destilação (2). A mistura é bombeada para o primeiro decantador (3) ou para o destilador (2), dependendo do tipo de processo e álcool adotados. Com a mistura no destilador, um sistema de aquecimento de óleo é fornecido para possibilitar a evaporação do excesso de álcool do estágio de reação, aumentando a cinética e a eficiência da reação. A bomba de vácuo (5) retira o oxigênio do destilador (2) e diminui o ponto de ebulição do álcool, evitando a oxidação e a consequente degradação do biodiesel. O excesso de álcool condensa e é recuperado no reservatório de álcool (6) e pode ser reutilizado. Depois da destilação, a mistura é bombeada (7) para o segundo decantador (8) para a separação de fases por gravidade. A fração pesada, a glicerina bruta, é enviada para a coluna de glicerina (9), e a porção leve, ésteres graxos (biodiesel bruto), passa através das colunas de lavagem (10 e 11) com a atuação da bomba de vácuo e do ar comprimido (5). O biodiesel bruto percola a resina de troca iônica que retém os resíduos, obtendo-se um biodiesel de alta pureza, que finalmente é enviado ao reservatório de biodiesel (12). 


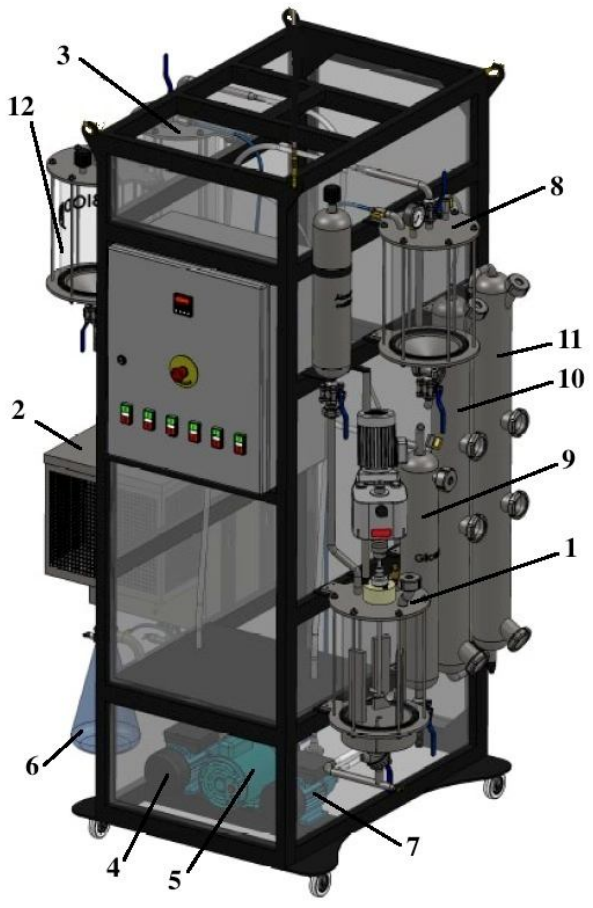

FIGURA 3 - Partes principais da planta de biodiesel

\section{Metodologia}

A planta, originalmente, trabalha como exposto anteriormente, i.e., em modo semiautomático. Não havia nenhuma válvula automática nem controle automatizado para as bombas. A indicação era realizada por medição direta nos instrumentos, e a abertura e fechamento das válvulas eram feitos manualmente. Embora a planta tivesse uma boa implementação do processo de produção do biodiesel e permitisse a obtenção de diferentes tipos de produtos, havia a falta de um sistema de controle automático. Então, a automação se deu em três níveis: o primeiro consistiu na especificação física e substituição dos dispositivos manuais, tais como válvulas atuadoras; o segundo nível, na automação do sistema de controle, realizada pela substituição dos dispositivos eletrônicos por um CLP (Controlador Lógico Programável) (BOLTON, 2009), que consiste em um tipo de computador para o controle do processo; e o terceiro, no desenvolvimento de um sistema supervisório, capaz de monitorar o processo através de uma interface gráfica computacional. Este último também permite o controle do processo pela internet. A FIG. 4 apresenta o novo arranjo de todo o sistema. Esse tipo de sistema integrado é também denominado Sistema SCADA (Supervisory Control and Data Acquisition ou Controle Supervisório e Aquisição de Dados) (BAILEY; WRIGHT, 2003) e é comumente adotado em plantas industriais.

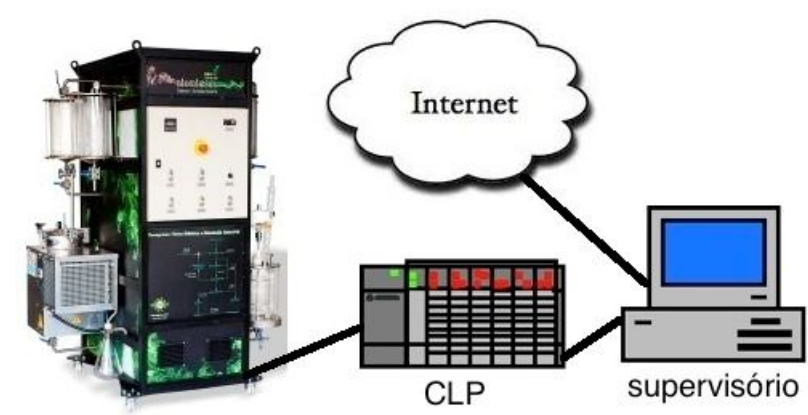

FIGURA 4 - Arquitetura do sistema.

\section{IMPLEMENTAÇÃO dA AUTOMAÇÃO}

Uma vez que os dispositivos do sistema original eram manuais, estes tiveram que ser substituídos por novos, os quais poderiam ser comandados automaticamente pelo CLP. Isso significa substituir as válvulas operadas manualmente por manejadas através de solenoides em todas as transições entre os diferentes vasos do sistema. Dessa forma, o fluido pode ser retido e liberado do reator para o destilador ou para o primeiro decantador, dependendo das escolhas operacionais, e pode ser direcionado automaticamente, através do CLP, sem a interferência do operador.

Do mesmo modo, as duas bombas de vácuo e o motor do compressor receberam soft starters, o que permitiu um melhor controle do acionamento e desligamento destes equipamentos. $O$ tanque de mistura foi modificado para receber um inversor de frequência, permitindo um melhor controle de velocidade da 
mistura. Ambos soft starters e o inversor são controlados pelo CLP.

Uma nova instrumentação foi implementada para permitir a transmissão da condição da planta ao CLP. Os valores em tempo real de nível, temperatura e pressão do processo podem agora ser vistos no sistema supervisório, que também gera condições de alarme, gráficos de tendência e históricos de log.

\subsection{ProgramaÇão do CLP}

A sequência e lógica dos eventos foram programadas de maneira a se converter os procedimentos anteriormente manuais em uma sucessão automática de comandos a serem executados pelo CLP (FONSECA; SEIXAS; BOTTURA, 2008). A FIG. 5 mostra a lógica do programa CLP resultante, utilizando representação SFC - Sequential Function Chart do IEC 61131-3.

O CLP foi programado utilizando-se o padrão internacional para controlador lógico programável IEC 61131-3, que permite melhor entendimento e documentação do programa, assim como aplicação das melhores práticas de programação de CLP.

O fluxo original dos procedimentos foi traduzido em uma sequência de passos, separados por equipamento (reator, decantador 1 e 2, destilador, colunas) e dispositivos principais, tais como bombas de vácuo. Todos foram programados usando linguagem ladder no CLP. O programa resultante é capaz de comandar a maioria dos dispositivos, como bombas e solenoides para abrir e fechar as válvulas automaticamente, o que traz a automatização à planta anteriormente manual. As variáveis de processo, tais como temperatura e tempo, também são controladas pelo computador.

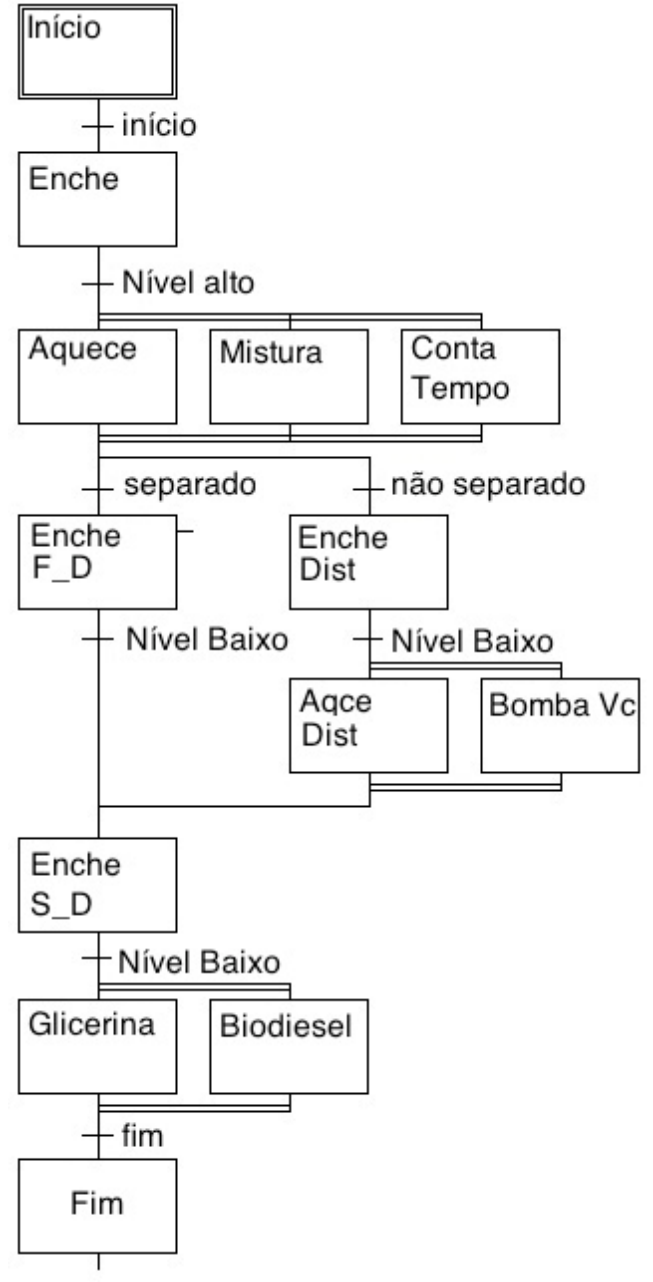

FIGURA 5 - Automação do processo

\subsection{SISTEMA SUPERVISÓRIO}

O sistema supervisório é um tipo de software que possibilita o controle e a supervisão de um processo automatizado (BOYER, 2009). Ele contém telas gráficas para mostrar o status do sistema ao operador e permite sua interação com a planta controlada através de uma IHM (Interface Homem-Máquina). O operador é apto a ajustar e mudar set points, ler os valores das variáveis de processo e tomar decisões operacionais em tempo real.

O sistema supervisório tem uma camada de comunicação que permite a troca de dados com o CLP. Desse modo, o sistema supervisório pode enviar 
e receber dados ao CLP, que está controlando a planta.

Foi implementada uma tela para cada equipamento controlado, além de telas adicionais para dados históricos e alarmes da planta (ELIPSE, 2008).

Outra tela de ajuste de receitas foi instalada de modo que diferentes tipos de óleos vegetais podem ser selecionados, uma vez que a temperatura e o tempo do processo podem variar em função do produto a ser processado.

A FIG. 6 apresenta a tela do reator com as variáveis principais a serem controladas. O nível do tanque e sua temperatura são medidos em tempo real, assim como o estado dos solenoides (aberto ou fechado) que controlam as válvulas de entrada e saída.

O operador pode alterar alguns parâmetros, como a rotação do agitador, o tempo total de reação e o set point de temperatura, através dessa tela.

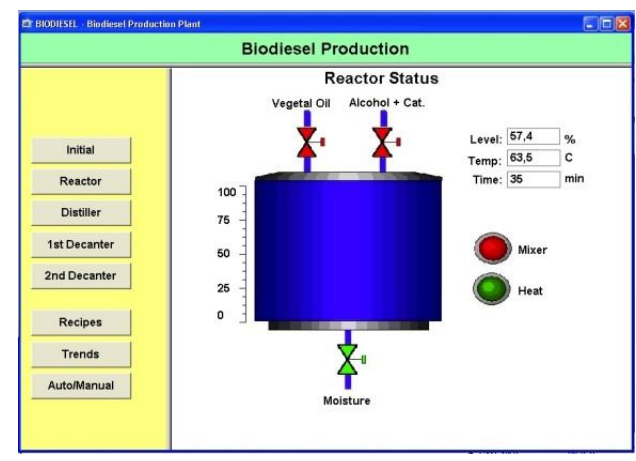

FIGURA 6 - Tela do reator

\subsection{Sistema e Rede}

O supervisório utilizado foi o Elipse E3, um supervisório brasileiro que roda sobre o sistema operacional Microsoft Windows 7. O supervisório é conectado a um CLP Siemens S7-1200 por uma rede Profinet (POPP, 2007). O sistema também tem acesso à intranet, que, por sua vez, está conectada à internet.
Então, utilizando-se um servidor web, o supervisório é capaz de colocar suas telas na rede.

\section{Resultados e Discussão}

Os primeiros resultados demonstram uma melhor maneira de se controlar a planta, diferentemente da planta original, que é semiautomática. O processo controlado por computador é mais confiável, uma vez que elimina muitas possibilidades de falhas operacionais que poderiam levar à perda de produto.

Isso é relevante, visto que a planta é utilizada no meio acadêmico, por vários tipos diferentes de usuários, desde professores a estudantes, e a operação manual se torna uma possível fonte de erro de uso.

Um melhor controle de temperatura se mostrou importante. O termostato original não era tão estável quanto o implementado no CLP. Isso faz com que todo o ciclo seja mais previsível e afeta sobremaneira a qualidade final do produto.

O CLP também controla melhor o misturador do que o controlador original, que era mecânico e não permitia uma boa faixa de atuação, o que, algumas vezes, causava um acúmulo de material nas paredes do reator.

Este trabalho propôs e implementou a automação da produção de biodiesel objetivando dois aspectos distintos: o primeiro foi a promoção de um melhor controle do ciclo de produção de biodiesel, de modo a obter um produto de maior qualidade; e o segundo, aplicar a técnica de automação numa planta existente, respeitando os padrões atuais de engenharia e criando um produto de automação de alta qualidade ao final. Mesmo que algumas modificações ainda possam ser introduzidas ao sistema, os objetivos foram alcançados, de acordo com o que se comprovou em todos os testes de estabilidade do sistema e de qualidade do produto final. 


\section{CONCLUSÕES}

Pode-se concluir que o uso da automação em plantas existentes pode elevar a produção dessas plantas a um nível mais alto de qualidade e confiabilidade. O retrofitting do sistema existente é um campo inteiro de aplicação da automação, e não somente de plantas novas.

Para fazê-lo, de fato, deve-se ter um bom entendimento do processo de produção, o que pode ser a dificuldade real e um desafio na maneira de se ter uma boa implementação do sistema computacional.

O propósito de tal empreendimento reside na discussão sobre a busca de novas fontes de energia que poderiam ser confiáveis e limpas, levando à necessidade do entendimento não somente de um tipo de tecnologia ou outra, como automação ou produção de biodiesel, mas o entendimento do problema como um todo, que se mostra um desafio aos engenheiros e à Engenharia.

Este objetivo somente pode ser alcançado pela interação de diferentes atores envolvidos e pela multidisciplinaridade que leva ao envolvimento de diferentes pessoas, áreas e conhecimentos.

Dada a importância da busca pela eficiência e a clara necessidade de melhoria dos meios de produção do biodiesel nacional, este trabalho demonstra que o caminho da automação do processo de produção de biodiesel pode ser uma das soluções para a obtenção de um produto melhor, sem que isso se traduza em um grande impacto sobre plantas atualmente operacionais.

\section{REFERÊNCIAS}

BAILEY, D.; WRIGHT, E. Practical SCADA for Industry. Newnes, 2003. 304 p.

BIOMINAS. Manual de Instalação e Operação: Usina de Biodiesel Didática e Simulação Industrial, 2012. $48 \mathrm{p}$.

BOLTON, W., Programmable Logic Controllers, Fifth Edition, Newnes, 2009. 416 p.

BOYER, S. A. Scada: Supervisory Control And Data Acquisition. ISA: The Instrumentation, Systems, and Automation Society, 2009. 257 p.

DRAPCHO, C.; NGHIEM, J.; WALKER, T. Biofuels Engineering Process Technology. McGraw-Hill, 2008. $371 \mathrm{p}$.

ELIPSE. Tutorial Elipse E3, versão 3. Elipse Software Ltda, São Paulo, 2008. 168 p.
FONSECA, M. O.; SEIXAS, C.; BOTTURA, J. A. Aplicando a Norma IEC 61131 na automação de Processos. São Paulo: ISA Distrito 4, 2008. 568 p.

MINISTÉRIO DE MINAS E ENERGIA, Programa Nacional de Produção e Uso do Biodiesel. Disponível em:

http://www.mme.gov.br/programas/biodiesel/menu/bio diesel/pnpb.html. Acesso: 10/07/2013.

POPP, M. Industrial Communication with PROFINET, Karlsruhe, Germany, 2007. 385 p. 